\title{
From the Somigliana waves to the evanescent waves
}

\author{
Pietro Caloi
}

Annali di Geofisica, Vol. 25, n. 4, 1972.

\section{ABSTRACT}

The Rayleigh equation has real coefficients; therefore, also the case of complex conjugated roots may be explained physically. The Author proves that the Somigliana waves may be formed for Poisson ratio values until 0.30543; for gradually less rigid media, they are missing altogether and degenerate into evanescent waves.

1. In some previous notes [Caloi 1966a, 1966b, 1967, 1969] I have dealt with the physical interpretation of the roots of the Rayleigh equation which are above the unit, for the values of the $\sigma$ coefficient of Poisson to which correspond three real roots for the Rayleigh equation. And I have proved that those roots have an exact physical meaning: they permit the theoretical interpretation of sizable groups of seismic oscillations which I named waves of Somigliana. I found then the limits within which the Somigliana waves originate, within the real roots above the unit, and I emphasized how interesting it was to include the study of such waves into the research of stratifications building up the Earth's crust.

However, as was already noted by Somigliana in this third contribution to the propagation of seismic waves [Somigliana 1918], due to the homogeneity of equations of motion and to the fact that the Rayleigh equation has real coefficients, also the case of complex, conjugated roots may be explained physically with the separation of the real from the imaginary part of roots.

This is what I am undertaking as follows.

2. First of all, let us try to find analytically the value of $\sigma$ separating the real field from the complex field for roots above the unit.

This value has already been obtained empirically in the previous note [Caloi 1969].

As is known, the Rayleigh equation is expressed in its most known form with the usual meaning of symbols [Caloi 1966a]:

$$
\left(2-\frac{v_{3}^{2}}{v_{2}^{2}}\right)^{4}=16\left(1-\frac{v_{3}^{2}}{v_{1}^{2}}\right)\left(1-\frac{v_{3}^{2}}{v_{2}^{2}}\right)
$$

from which, after having made [Caloi 1966a]

$$
v_{3}^{2}=\chi v_{2}^{2},
$$

follows

$$
\chi^{3}-8 \chi^{2}+8\left(3-2 \frac{v_{2}^{2}}{v_{1}^{2}}\right) \chi-16\left(1-\frac{v_{2}^{2}}{v_{1}^{2}}\right)=0
$$

Remembering that $\frac{v_{2}^{2}}{v_{1}^{2}}=\frac{1-2 \sigma}{2(1-\sigma)}$, we put

$$
\varepsilon=1-\frac{v_{2}^{2}}{v_{1}^{2}}=\frac{1}{2(1-\sigma)} ;
$$

whence (1) becomes

$$
\chi^{3}-8 \chi^{2}+8(1+2 \varepsilon) \chi-16 \varepsilon=0 .
$$

Let us see how the roots of this equation vary when $\sigma$ varies between 0 and $\frac{1}{2}$, that is for

$$
\frac{1}{2} \leqslant \varepsilon \leqslant 1 .
$$

Now we free (2) from the second degree term in $\chi$. To this end we put

$$
\chi=\delta+\frac{8}{3} .
$$

Equation (2) changes then into

$$
\delta^{3}+\frac{8}{3}(6 \varepsilon-5) \delta+\frac{16}{3}\left(5 \varepsilon-\frac{28}{9}\right)=0 .
$$

We make

$$
\frac{8}{3}(6 \varepsilon-5)=p, \frac{16}{3}\left(5 \varepsilon-\frac{28}{9}\right)=q
$$

thus obtaining

$$
\delta^{3}+p \delta+q=0 .
$$

If $p$ and $q$ are real, we know from the mathematical analysis that the condition for the three roots of (3) to be real is expressed in the relation

$$
\frac{q^{2}}{4}+\frac{p^{3}}{27} \leqslant 0 .
$$

Considering the values of $p$ and $q$, we have in our case 


$$
\frac{q^{2}}{4}+\frac{p^{3}}{27} \frac{4^{3}}{3^{6}}\left[(45 \varepsilon-28)^{2}+(12 \varepsilon-10)^{3}\right] .
$$

For the roots to be real, we must have

$$
\Delta(\varepsilon)=(45 \varepsilon-28)^{2}+(12 \varepsilon-10)^{3} \leqslant 0 .
$$

It is easily found that the value of $\varepsilon$ which annulls $\Delta(\varepsilon)$ is

$$
\varepsilon=0.6785
$$

And since

$$
\sigma=1-\frac{1}{2} \cdot \frac{1}{\varepsilon}
$$

it follows

$$
\sigma=0.26308
$$

which practically coincides with the value previously obtained $(\sigma=0,26305)$. It is in correspondence to this value that the two roots above the unit of the Rayleigh equation coincide; in fact one obtains $\chi_{2,3}=3.5754$ [Caloi 1969].

Therefore, in order to have a real root above the unit and two complex conjugated roots, it must be

$$
\frac{q^{2}}{4}+\frac{p^{3}}{27}>=0
$$

to which corresponds for $\sigma$ the field of variability:

$$
0.26305<\sigma \leqslant 0.5
$$

3. There remained now to calculate a series of complex, conjugated root couples for $\sigma$ values within the above limits.

Calculations have been made for the following $\sigma$ values: $0.265 ; 0.27 ; 0.30 ; 0.305 ; 0.35 ; 0.40 ; 0.50$.

The Rayleigh equations pertinent to the above $\sigma$ values are:

for $\sigma=0.2653 .1277 \chi^{3}-25.022 \chi^{2}+59.065 \chi-34.043=0$

for $\sigma=0.27 \quad 3.1739 \chi^{3}-25.391 \chi^{2}+60.174 \chi-34.782=0$

for $\sigma=0.3 \quad 3.50 \quad \chi^{3}-28.0 \quad \chi^{2}+68.0 \quad \chi-40.0 \quad=0$

for $\sigma=0.3053 .564 \chi^{3}-28.512 \chi^{2}+69.536 \chi-41.024=0$

for $\sigma=0.35 \quad 4.333 \quad \chi^{3}-34.66 \quad \chi^{2}+88.0 \quad \chi-53.33=0$

for $\sigma=0.4 \quad 3 \quad \chi^{3}-24 \quad \chi^{2}+64 \quad \chi-40 \quad=0$

for $\sigma=0.5 \quad \chi^{3}-8 \quad \chi^{2}+24 \quad \chi-16 \quad=0$

The corresponding roots are (Table 1):

\begin{tabular}{lll}
$\sigma$ & \multicolumn{1}{c}{$\chi_{1}$} & \multicolumn{1}{c}{$\chi_{2,3}$} \\
\hline 0.265 & 0.8498 & $3.5752 \pm i 0.16221$ \\
0.27 & 0.85125 & $3.5743 \pm i 0.3120$ \\
0.3 & 0.86009 & $3.5714 \pm i 0.7284$ \\
0.305 & 0.86154 & $3.5690 \pm i 0.7896$ \\
0.35 & 0.8740 & $3.5625 \pm i 1.1791$ \\
0.4 & 0.8877 & $3.5562 \pm i 1.5406$ \\
0.5 & 0.9128 & $3.5436 \pm i 2.2302$
\end{tabular}

4. I asked myself whether all $\sigma$ values in the interval $0.26305 \div 0.5$, were leading to roots to which correspond Somigliana waves. Those roots are complex and conjugated and some of their values have been given under 3. They are complex, hence also the values of [Caloi 1966a, 1966b],

$$
v_{3}=v_{2} \sqrt{\chi}, \operatorname{tang}^{2} e_{1}=\frac{v_{2}^{2}}{v_{1}^{2}} \chi-1, \operatorname{tang}^{2} e_{2}=\chi-1 .
$$

Let us indicate a general complex root as follows

$$
\chi_{2,3}=r+i c
$$

The formulas (10) will then be written

$$
\begin{aligned}
& \operatorname{tang}^{2} e_{1}=r \frac{v_{2}^{2}}{v_{1}^{2}}-1+i c \frac{v_{2}^{2}}{v_{1}^{2}}, \\
& \operatorname{tang}^{2} e_{2}=r-1+i c, v_{3}=v_{2} \sqrt{r+i c} .
\end{aligned}
$$

The relation

$$
R+i C=\sqrt{r+i c},
$$

allows to obtain

$$
R=\left(\frac{\sqrt{r^{2}+c^{2}}+r}{2}\right)^{1 / 2}, C=\left(\frac{\sqrt{r^{2}+c^{2}}-r}{2}\right)^{1 / 2}
$$

After the value of $\sigma$, included in the above interval, has been assigned, the Rayleigh equation furnishes the corresponding couple of complex, conjugated roots. Thus $r$ and $c$ are obtained and thence tang $e_{1}$, tang $e_{2}$ and $v_{3}$ pertaining to the chosen $\sigma$ value.

After separating the real part from the imaginary one, we can thus arrive at the real values of $e_{1}$ (if existing), of $e_{2}$ and of $v_{3}$. Let us indicate the latter

while putting

$$
v_{3}^{\prime}=v_{2} R
$$

$$
v_{3}=v_{2} C
$$

where the negative value for $C$ is being taken.

On the basis of the $r$ and $c$ values taken from the previous Table 1 we obtain Table 2 by varying $\sigma$ as follows:

\begin{tabular}{lllllll}
$\sigma$ & $\mathrm{v}_{2}^{2} / \mathrm{v}_{1}^{2}$ & $\operatorname{tang}^{2} e_{1}$ & $e_{1}$ & $\operatorname{tang}^{2} e_{2}$ & $e_{2}$ & $R$ \\
\hline 0.265 & 0.31973 & 0.14310 & $20^{\circ} 45^{\prime} .25$ & 2.5752 & $58^{\circ} 04^{\prime} .25$ & 1.8913 \\
0.27 & 0.31507 & 0.12615 & $19^{\circ} 33^{\prime}$ & 2.5743 & $58^{\circ} 04^{\prime}$ & 1.8924 \\
0.3 & 0.2857 & 0.02035 & $8^{\circ} 07^{\prime}$ & 2.5714 & $58^{\circ} 03^{\prime}$ & 1.8995 \\
0.305 & 0.2805 & 0.0011045 & $1^{\circ} 51^{\prime}$ & 2.5690 & $58^{\circ} 02^{\prime}$ & 1.9006 \\
0.35 & 0.23077 & -0.1779 & - & 2.5625 & $58^{\circ} 00^{\prime}$ & 1.9125 \\
0.4 & 0.16667 & -0.4073 & - & 2.5562 & $57^{\circ} 58^{\prime} .5$ & 1.9277 \\
0.5 & 0 & -1 & - & 2.5436 & $57^{\circ} 55^{\prime}$ & 1.99605
\end{tabular}




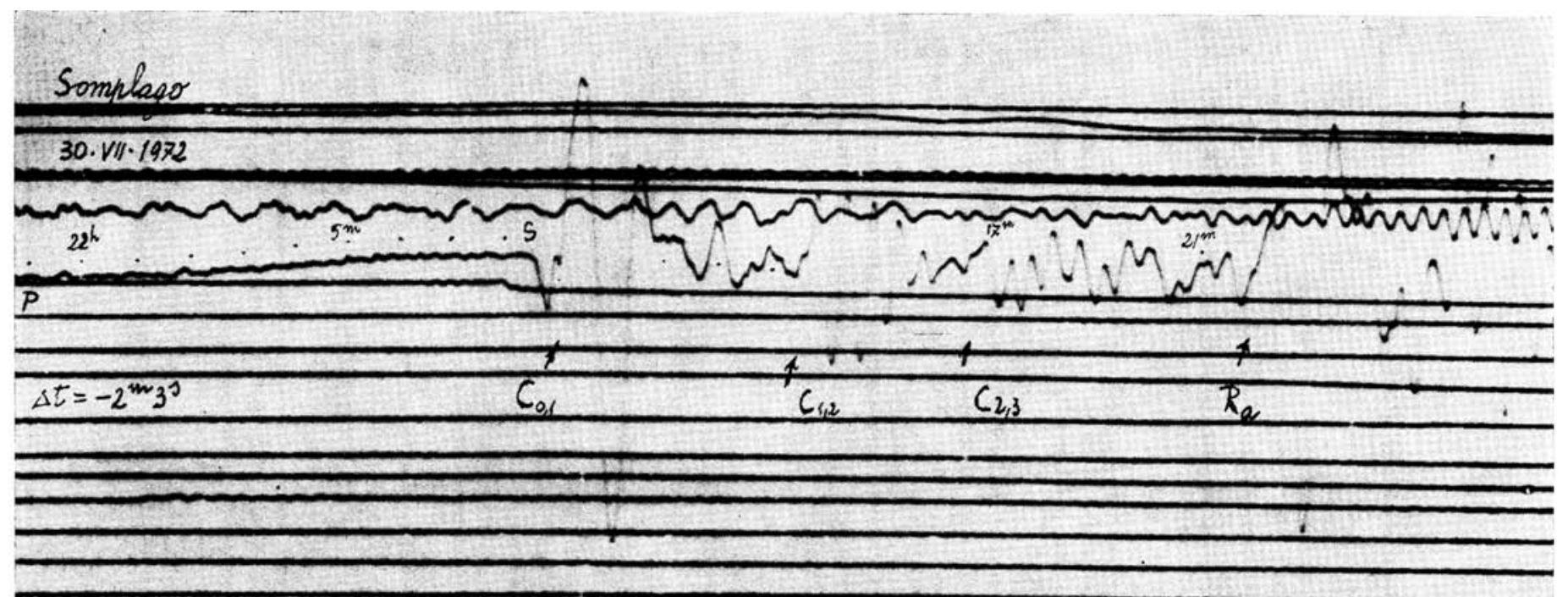

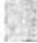

Figure 1. Examples of $C_{0,1}\left(T=688 \mathrm{ab}\right.$.), $C_{1,2}\left(T=34^{\mathrm{s}} \mathrm{ab}\right.$. $)$ and $C_{2,3}\left(T=23^{\mathrm{s}} \mathrm{ab}\right.$.) waves - by the author generically indicate as Somigliana waves - record at Somplago (on the Cavazzo Lake) by a seismograph, with free period of about $120^{\mathrm{s}}$ and optical magnification, in occasion of Alaska earthquake of July $30,1972\left(57^{\circ}, \mathrm{ON}-135^{\circ} .9 \mathrm{~W}: H=21.45 .11,1 \mathrm{GMT}: h=10 \mathrm{~km}: M=7.8\right)$ at an epicentral distance of about $8350 \mathrm{kms}$. For large earthquake (as this Alaska earthquake) $C_{0,1}$ waves can affect the outer layer of mantle, from the top of the astenosphere (low-velocity channel) to the Earth's surface (thickness of about $70 \mathrm{kms})$.

It follows from the analysis of the Table 2 that the Somigliana waves may vary for $\sigma$ values until 0.31 ; for gradually less rigid medium they are missing altogether and degenerate into ordinary transversal waves. The values of efficient angles, for longitudinal incidence, presuppose incidences nearing rapidly the right angle. So far as transversal incidence is concerned, the efficient angles increase slightly as the rigidity of the medium decreases and reach a limit angle of incidence of about $32^{\circ}$ to which corresponds the total reflection.

Considering as well the complex conjugated roots of the Rayleigh equation, the efficient angles bringing about Somigliana waves are the ones corresponding to the roots for the following field of variability of $\sigma$.

$$
0<\sigma<0.31 \text {, }
$$

although, practically [Caloi 1969], this is reducing to

$$
0.25<\sigma<0.31 \text {. }
$$

Anyhow, the use of complex conjugated roots indicates an enlargement and the limit of the field of variability of the Poisson coefficient.

Concerning the propagation velocity of the Somigliana waves (if existing), it is noted that it increases as a increases (that is as rigidity decreases) and reaches the maximum value

$$
v_{3}^{\prime}=1.9006 \cdot v_{2}
$$

for the limit value of $\sigma=0,305$.

If in the expressions of $u_{1}, u_{2}, w_{1}, w_{2}$ [Caloi 1969] we put $\Phi\left(x-v_{3} t\right)$ under power form

$$
\Phi\left(e-v_{3} t\right)=e^{i p\left(x-v_{3} t\right)},
$$

and observing that $v_{3}=v_{3}^{\prime}+i v_{3}$, we will have

$$
\Phi\left[x-\left(v_{3}^{\prime}+i v_{3}\right) t\right]=e^{i p\left(x-v_{3} t\right)} \cdot e^{p v_{3} t},
$$

where $p$ indicates the pulsation of the oscillation and $v_{3}$ is a negative constant which may be considered as the extinction coefficient of the oscillation in time.

For $\sigma=0.265$, for instance, $v_{3}=-0.043012 \cdot v_{2}$, and for $\sigma$ $=0.27, v_{3}=-0.08277 \cdot v_{2} ;$ whereas for $\sigma=0.305$, we have $v_{3}=$ $-0.2076 \cdot v_{2}$. Therefore, at equal frequencies the propagation of the Somigliana wave is extinguished more quickly as rigidity decreases.

5. Let us have a closer look how the Somigliana waves change when their propagation is in elastic media whose $\sigma$ coefficient shows a trend toward the value of 0.30543 .

Let us consider the case of transversal incidence, where we have

$$
\operatorname{tang} e_{1}=-\left(\chi \frac{v_{2}^{2}}{v_{1}^{2}}-1\right)^{1 / 2}, \operatorname{tang} e_{2}=(\chi-1)^{1 / 2} .
$$

If $\Phi\left(x-v_{3} t\right)$ is not periodical, here applies the relation [Caloi 1969][Caloi 1969]

$$
\alpha_{1}=\frac{1}{2} \alpha_{2} \frac{\frac{u_{0}}{w_{0}}+1 /\left(\chi \frac{v_{2}^{2}}{v_{1}^{2}}-1\right)^{1 / 2}}{\frac{u_{0}}{w_{0}}+(\chi-1)^{1 / 2}}(2-\chi) .
$$

$\chi$ always differs from 2 . 
The following Table 3 is valid:

$$
\sigma \quad\left(\chi \frac{v_{2}^{2}}{v_{1}^{2}}-1\right)^{1 / 2} \quad(\chi-1)^{1 / 2}
$$

\begin{tabular}{lll}
\hline 0.26305 & 0.3868 & 1.6048 \\
0.265 & 0.3783 & 1.60475 \\
0.27 & 0.3552 & 1.60445 \\
0.3 & 0.14265 & 1.60355 \\
0.305 & 0.033235 & 1.6028 \\
0.30543 & 0.0 & 1.6028 \\
0.4 & imaginary & 1.5988
\end{tabular}

As $\sigma$ tends toward the value $0.30543, \alpha_{1}$ is thus tending toward the infinite. Now, in the expressions of $u_{0}, w_{0}$ - see (1) of Caloi $1969-, \alpha_{1}$, the quantity characterizing the longitudinal component, acts as a denominator in the relative terms.

Therefore, these annull each other, namely the contribution of the longitudinal wave in the formation of the Somigliana wave falls away, and this degenerates into a trasversal wave.

However for $\sigma=0.30543$, the efficient angle of incidence of the transversal waves, is $31^{\circ} 57^{\prime} .4$, which coincides with the angle of total reflection of the incident transversal wave. In fact is:

$$
\frac{v_{2}^{2}}{v_{1}^{2}}=\frac{1-2 \sigma}{2(1-\sigma)}=0.28013 \text {; }
$$

so that

$$
\sin i_{2}=\frac{v_{2}^{2}}{v_{1}^{2}}=0.52927,
$$

which gives for $i_{2}$ the above value.

Therefore, the efficient angle for transversal waves incident at the intersurface with the outer stratification, approximates in less rigid media the angle of incidence to which corresponds the total reflection, which is reached, as could be seen, for $\sigma=0.30543$. Hence, in the field where $\sigma$ varies from 0.30543 to 0.5 there are no Somigliana waves, since for them the total reflection of the incident transversal waves takes place.

The formation of Somigliana waves requires a physically finite medium beyond the surface which is hit by the wave coming from an indefinite medium [Caloi 1967]. When the longitudinal reflected wave vanishes as progressive ordinary wave, for satisfying the conditions at the intersurface it is necessary to introduce an evanescent wave. If we indicate the transversal reflected wave (oscillating, of course, in the principal plane) by

$$
\psi=e^{k z} \sin (\omega t-\alpha x)
$$

we will have

$$
\begin{aligned}
& u=\frac{\delta \psi}{\delta x}=-\alpha e^{k z} \sin (\omega t-\alpha x) \\
& w=\frac{\delta \psi}{\delta x}=-k e^{k z} \sin (\omega t-\alpha x)
\end{aligned}
$$

where $u$ and $w$ are the horizontal and vertical motion components.

The resultant of these movements, however, is the socalled evanescent wave which - as the Rayleigh waves - forces the reached particle to describe an elliptical motion [Caloi 1955 , pages $300-304]$. In the variability field

$$
0.30543 \leqslant \sigma \leqslant 0.5
$$

which means that in always more incompressible media the Somigliana waves (possible only by transversal incidence) degenerate into evanescent waves.

In conformity to what happens in the propagation of the light, the velocity of evanescent waves in the second medium is $v_{2} / \sin i_{2}$, where $i_{2}$ is the angle of incidence. Practically it coincides, therefore, with $R v_{2}$ where $R$ is to be taken from the Table 2 . In case of the limit angle of incidence $\left(i_{2}=31^{\circ} 58^{\prime}\right)$, is in fact $v_{2} / \sin i_{2}=1.8894 v_{2}$, equal - at lower than $1 / 1000$ - to the value of $v_{3}^{\prime}$.

\section{References}

Caloi, P. (1955). Ci,j, Annali di Geofisica, VIII, 3, 293-313. Caloi, P. (1966a). L'equazione di Rayleigh e le onde di Somigliana. I: La teoria delle onde di Rayleigh, Atti Acc. Naz. dei Lincei, Classe Scienze fis. mat. e nat., XLI, ser. VIII, 1-2 Ferie.

Caloi, P. (1966b). L'equazione di Rayleigh e le onde di Somigliana. II: La teoria di Somigliana: rettifiche, conseguenze, Atti Acc. Naz. dei Lincei, Classe Scienze fis. mat. e nat., XLI, ser. VIII, 5.

Caloi, P. (1967). L'equazione di Rayleigh e 1e onde di Somigliana. III: Le Ci,j sono onde di Somigliana. Loro importanza per lo studio della crosta terrestre, Atti Acc. Naz. dei Lincei, Classe Scienze fis. mat. e nat., XLIII, ser. VIII, 6.

Caloi, P. (1969). L'equazione di Rayleigh e le onde di Somigliana. IV: Limiti d'insorgenza delle onde di Somigliana: loro esclusiva formazione nel piano principale, Atti Acc. Naz. dei Lincei, Classe Scienze fis. mat. e nat., XLVI, ser. VIII, 1.

Somigliana, C. (1918). Sulla propagazione delle onde sismiche. Nota III, Atti Acc. Naz. dei Lincei, Classe Scienze fis. mat. e nat., XXVII, ser. V, 1.

(C) 2010 by the Istituto Nazionale di Geofisica e Vulcanologia. All rights reserved. 